\title{
Date of Last Contact
}

National Cancer Institute

\section{Source}

National Cancer Institute. Date of Last Contact. NCI Thesaurus. Code C156916.

The date of the last contact. 